Article

\title{
Development and Application of an Additively Manufactured Calcium Chloride Nebulizer for Alginate 3D-Bioprinting Purposes
}

\author{
Lukas Raddatz $^{1,2}\left(\right.$, Antonina Lavrentieva ${ }^{1}$, Iliyana Pepelanova ${ }^{1}$, Janina Bahnemann ${ }^{1}{ }^{\circledR}$, \\ Dominik Geier ${ }^{2}$, Thomas Becker ${ }^{2}$, Thomas Scheper ${ }^{1}$ and Sascha Beutel ${ }^{1, *}$ \\ 1 Institut für Technische Chemie, Leibniz Universität Hannover, Callinstraße 5, 30167 Hannover, Germany; \\ raddatz@iftc.uni-hannover.de (L.R.); lavrentieva@iftc.uni-hannover.de (A.L.); \\ pepelanova@iftc.uni-hannover.de (I.P.); jbahnemann@iftc.uni-hannover.de (J.B.); \\ scheper@iftc.uni-hannover.de (T.S.) \\ 2 Lehrstuhl für Brau- und Getränketechnologie, Technische Universität München, Weihenstephaner Steig 20, \\ 80354 Freising, Germany; dominik.geier@tum.de (D.G.); tb@tum.de (T.B.) \\ * Correspondence: beutel@iftc.uni-hannover.de; Tel.: +49-511-762-2868
}

Received: 24 August 2018; Accepted: 5 November 2018; Published: 9 November 2018

check for updates

\begin{abstract}
Three-dimensional (3D)-bioprinting enables scientists to mimic in vivo micro-environments and to perform in vitro cell experiments under more physiological conditions than is possible with conventional two-dimensional (2D) cell culture. Cell-laden biomaterials (bioinks) are precisely processed to bioengineer tissue three-dimensionally. One primarily used matrix material is sodium alginate. This natural biopolymer provides both fine mechanical properties when gelated and high biocompatibility. Commonly, alginate is $3 \mathrm{D}$ bioprinted using extrusion based devices. The gelation reaction is hereby induced by a $\mathrm{CaCl}_{2}$ solution in the building chamber after material extrusion. This established technique has two main disadvantages: (1) $\mathrm{CaCl}_{2}$ can have toxic effects on the cell-laden hydrogels by oxygen diffusion limitation and (2) good printing resolution in the $\mathrm{CaCl}_{2}$ solution is hard to achieve, since the solution needs to be removed afterwards and substituted by cell culture media. Here, we show an innovative approach of alginate bioprinting based on a $\mathrm{CaCl}_{2}$ nebulizer. The device provides $\mathrm{CaCl}_{2}$ mist to the building platform inducing the gelation. The necessary amount of $\mathrm{CaCl}_{2}$ could be decreased as compared to previous gelation strategies and limitation of oxygen transfer during bioprinting can be reduced. The device was manufactured using the MJP-3D printing technique. Subsequently, its digital blueprint (CAD file) can be modified and additive manufactured easily and mounted in various extrusion bioprinters. With our approach, a concept for a more gentle 3D Bioprinting method could be shown. We demonstrated that the concept of an ultrasound-based nebulizer for $\mathrm{CaCl}_{2}$ mist generation can be used for 3D bioprinting and that the mist-induced polymerization of alginate hydrogels of different concentrations is feasible. Furthermore, different cell-laden alginate concentrations could be used: Cell spheroids (mesenchymal stem cells) and single cells (mouse fibroblasts) were successfully 3D printed yielding viable cells and stable hydrogels after $24 \mathrm{~h}$ cultivation. We suggest our work to show a different and novel approach on alginate bioprinting, which could be useful in generating cell-laden hydrogel constructs for e.g., drug screening or (soft) tissue engineering applications.
\end{abstract}

Keywords: 3D printing; bioprinting; alginate; bioink; cell culture technology; rapid tooling; hydrogels; customizable labware 


\section{Introduction}

One of the most important achievements of mankind is the invention of the printing by Johannes Gutenberg in $1450 \mathrm{AD}$. With this new method, the typesetting and printing of books was made easier, faster, and less expensive than ever before [1]. Book prices dropped and common people obtained access to literature, which is often referred to as the trigger for "democratization of knowledge" and paved the way for the Reformation and the European Enlightenment [2]. Today, about 570 years later, another disruptive technology revolutionizes our society: three-dimensional (3D) printing. Compared to conventional production strategies this technology enables the fabrication of complex structures in very short periods of time and at lower costs. Because of these advantages, it could, analogously to Gutenberg's invention, initiate the "democratization of manufacturing". Since its invention in the 1980s and after significant impact on the production industry, 3D printing currently finds application in biotechnological and chemical laboratories [3-7]. The current research generally confirms that it enables scientists to generate custom tissue constructs for cell screening. In the past years, the disadvantages of conventional two-dimensional (2D) cell culture for pharmaceutical research and industry have become more apparent, as accurate in vitro assays turn out to be of poor predictive value [8] caused by reduced cell-cell contacts in 2D cell cultivation. The extra cellular matrix (ECM) generation is limited significantly, resulting in completely different cell behavior compared to in vivo 3D tissues [9]. Bioprinting combines 3D printing technology with cell culture technology and it enables researchers to additively manufacture custom structures containing living cells and to imitate tissues [10]. The use of hydrogels as the biomaterials in 3D bioprinting has been described intensively and the most frequently used methods involve: laser-induced forward transfer (LIFT), inkjet printing (IP), and extrusion or dispensing based (EB) [11-13]. Despite its comparable low resolution of about 200-500 $\mu \mathrm{m}$ in the $\mathrm{x} / \mathrm{y}$ plane, EB's fabrication speed is significantly higher compared to other methods and is often described as the most promising among bioprinting techniques [11,14,15]. One main aspect of 3D bioprinting and bioink compositions are the physical properties of the tissue that shall be imitated-the spectrum reaches from hard cartilage and bone tissue [16] to super soft tissue. The latter was realized using cryogenic 3D printing methods and poly(vinyl) based bioinks. With this technique, constructs can be manufactured, which are of comparable softness as brain and lung tissue [17]. Besides hydrogels as bioinks, silicone compositions are frequently used, as well. Due to their biocompatibility, oxygen permeability, and porous structure, they are suitable for several applications in 3D bioprinting. However, despite its use for the mimicry of mechanical structures, its applicability is limited and e.g., not suitable for drug release or direct cell to cell studies [18]. Recent innovations led to the fabrication of a heart valve using an alginate based bioink [19], but it is still far away from generating transplantable tissues. On the other hand, functional human tissue for drug testing [20], collagen-derived natural leather, and food [21] are already purchasable-to list a few examples. The most frequently used bioprinting materials are hydrogels, which are water retaining insoluble polymers consisting of e.g., proteins and carbohydrates. The highly biocompatible substances, allow cell remodeling, attachment, and spreading. Ideally, the gel undergoes a quick and controlled sol-gel transition, through a change in temperature or $\mathrm{pH}$. Some hydrogels, however, have to be modified chemically to allow fast curing during or after 3D bioprinting. A high variety of materials are used for 3D bioprinting, among which gelatin methacrylate, collagen/gelatin, fibrinogen, agarose, methacrylated hyaluronic acid, and alginate play an important role. Also, compositions are used in order to improve cell-hydrogel interactions or printability [22]. Sodium alginate ( $\mathrm{Na} / \mathrm{Alg}$ ) based bioinks have already been extensively studied and numerous publications demonstrate their high potential and wide range of applicability. $\mathrm{Na} / \mathrm{Alg}$ is a salt derived from the alginic acid and forms a water insoluble hydrogel by the addition of divalent ions, such as $\mathrm{Ca}^{2+}$. It is biodegradable, non-toxic, non-immunogenic, and composed of linear polysaccharides (guluronic and mannuronic acids) [23]. This biopolymer gets utilized both as stand-alone material and as an additive for bioink compositions.

Several groups are using cell-laden alginate hydrogels containing primary hepatozytes, HUVECs, or human chondrocytes in order to manufacture respective tissue at some point [24-28]. 
Furthermore, microphysiological systems and vascular graft structures were successfully bioprinted and described $[25,29]$. Sodium alginate is polymerized by ionic crosslinks with $\mathrm{CaCl}_{2}$. Normally, in order to convert the liquid alginate to a gel, the cell-laden bioink is printed directly into a $\mathrm{CaCl}_{2}$ solution. Often, this extensive and complex-to-handle procedure leads to inadequate results. The post-processing, including construct transfer to a cultivation vessel, can lead to its destruction and can influence the cell viability. Moreover, oxygen transfer to the cells in the biomaterial is inhibited when $3 \mathrm{D}$ bioprinted directly into a $\mathrm{CaCl}_{2}$ solution. Other researchers are facing this issue by using alginate containing composites aiming to improve construct quality and hydrogel properties. "CELLINK" is a commercially available bioink that combines nanocellulose and alginate. This hydrogel has fast crosslinking features, making it relevant for soft tissue applications producing more stable objects $[10,23]$. In other approaches using gelatin [30] or chitosan as accessory agent successfully improved bioprinting results [30,31]. However, the mentioned studies use alginate composite bioink. Another advance was made establishing a different curing method. The freeform reversible embedding of suspended hydrogels (FRESH) termed method utilizes a secondary hydrogel (for example gelatin) functioning as a temporary support [32]. The support matrix contains $\mathrm{CaCl}_{2}$ inducing the gelation immediately. It could be shown that soft hydrogel scaffolds can be manufactured using FRESH [32], although major disadvantages include the low product resolution, and again, its destruction during post processing and gelatin wash off.

We have developed a novel crosslinking approach using a custom 3D printed $\mathrm{CaCl}_{2}$ nebulizer. This method allows for a decrease of $\mathrm{CaCl}_{2}$ concentration to avoid possible toxic effects on the cells and to improve construct grip to the culture plate surface. Current protocols use either a higher $\mathrm{CaCl}_{2}$ concentration, a significant longer incubation time of the constructs in the curing solution, or combinations of different polymers $[19,27,33]$. In this study, the nebulization chamber LR06 was designed using CAD software and was printed with a multi jet printer (MJP). The LR06 provides high mist density without affecting the bioprinting process utilizing a piezo element. By applying alternating voltage to the element, the crystal starts to oscillate. This movement is transferred to the above-placed $\mathrm{CaCl}_{2}$ solution inducing compression waves in the liquid. At the surface of the solution, the high kinetic energy of the waves leads to the nebulization of the liquid, yielding a mist. Two theories are discussed to explain the process: (1) The capillary waves theory postulates that the nebulization takes place at the crests of instable waves at the liquid surface. According to (2), the cavitational bubble theory, a different effect is responsible for the nebulization: Caused by pressure differences between the compression waves, small gas bubbles deploy and implode, generating the mist [34-38]. The exact nebulization mechanism is yet not understood entirely [35]. Different concentrations of alginate were characterized in terms of viscosity and stiffness after polymerization. We successfully 3D bioprinted adipose-derived mesenchymal stem cells and murine fibroblasts (NIH-3T3-GFP) suspended in different alginate concentrations. The cells remained viable and the hydrogels stable during cultivation time. We successfully combined the nebulizer with the Allevi (Allevi Inc., Philadelphia, PA, USA) bioprinter Biobot 1 . By reason of its customizable building plate-mount, the LR06 can easily be adjusted to other bioprinter models. The therefore needed CAD .stl blueprints are provided and are ready to download as supplementary material (Files S1 and S2).

\section{Results}

\subsection{Design, Manufacturing and Functioning of the $\mathrm{CaCl}_{2}$ Nebulizer}

The presented 3D bioprinting system is a novel development and uses nebulized $\mathrm{CaCl}_{2}$ in order to induce $\mathrm{Na}$ / Alg polymerization reaction. The main parts of the system (lid and mist chamber with petri dish mount, displayed in grey, Figure 1) were designed using CAD software and additively manufactured with a MJP 3D printer. Using this method, high definition products can be manufactured in short periods of time and of high quality material. The printing material (epoxy resin) is further discussed in Section 2.1.1. The device was designed for the Allevi 1 bioprinter, but it can be modified in 
CAD and adapted for other extrusion-based bioprinters. The second part of this device is the nebulizer, mounted to the nebulizer adapter (Figure 1). For the mist generation, it uses a piezo element (piezo crystal). For the whole experimental set-up and workflow, Figure $1 \mathrm{C}$ provides the hyperlink to a demonstration video or see supplementary material (Video S1).

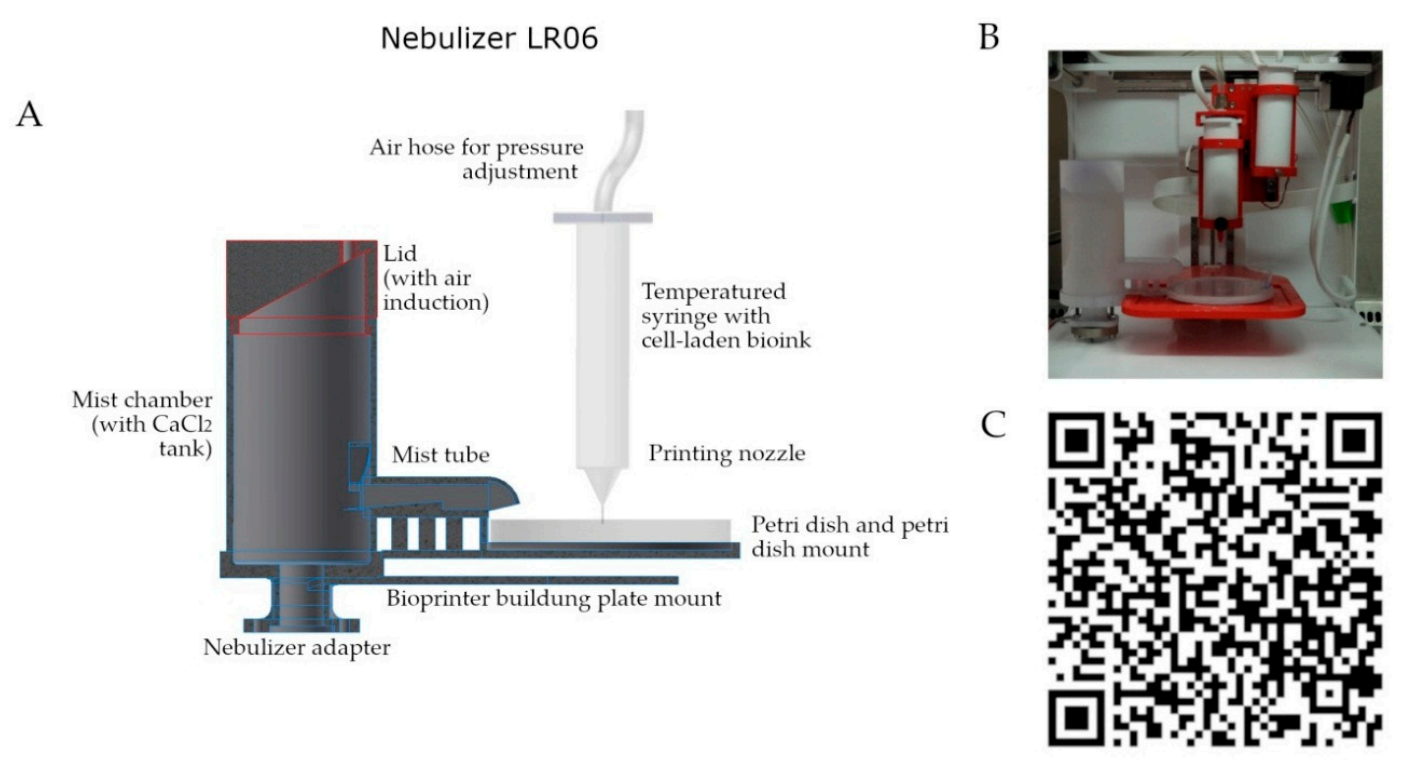

Figure 1. Additive manufactured $\mathrm{CaCl}_{2}$ Nebulizer LR06. (A) Schematic representation of the LR06 and extruder nozzle; (B) Photograph of the LR06 mounted to a bioprinter; (C) A demonstration video of the LR06 can be found in the supplementary section of the journal and linked as a QR-Code.

\subsubsection{Toxicity Evaluation of the Printing Material and Nebulization Effect}

The here demonstrated 3D printed nebulizer is made from a resin polymer [39] and it has to fulfill important characteristics, like chemical stability. The printing technique was already evaluated in previous studies, as were the fabricated materials for potential leaching of the cured resin when in contact with biological compounds. It could be shown that the use of this material has no negative influence on the cell viability of animal cell lines (L-929 and NIH-3T3) and cell growth of microbial cells (E. coli) [4]. During the ultrasound-driven nebulization process, the liquid is exposed to a high amount of kinetic energy. Material debris could reach the 3D printing platform carried by the mist. In order to evaluate the potential toxic effects on the cell culture, a cytotoxicity assay was performed. Therefore, conventional cell culture media (alpha-MEM) was nebulized both in the LR06 and a second (non-3D-printed) vessel of high performance polyoxymethylene (POM) plastic. The nebulization took place with complete reflux to the tank. This media was further used for a cultivation (mesenchymal stem cells) and a cell viability assay (CellTiterBlue ${ }^{\circledR}$ ). With this set-up, the cell viability of the cells cultivated in (1) the native media (control), (2) the nebulized media (without risk of debris contamination), and (3) the nebulized media in the LR06 can be evaluated. The results of the cell viability assay are shown in Figure 2 . The cell viability of both samples is distinctively above 90\% to control, indicating no negative effect on the cells (referring to ISO-10993-5:2009) [40]. The slight decrease of $1 \%(\mathrm{POM}$ ) and $6.9 \%$ (LR06) in cell viability as compared to the control notable in Figure 2 could not be statistically validated. 


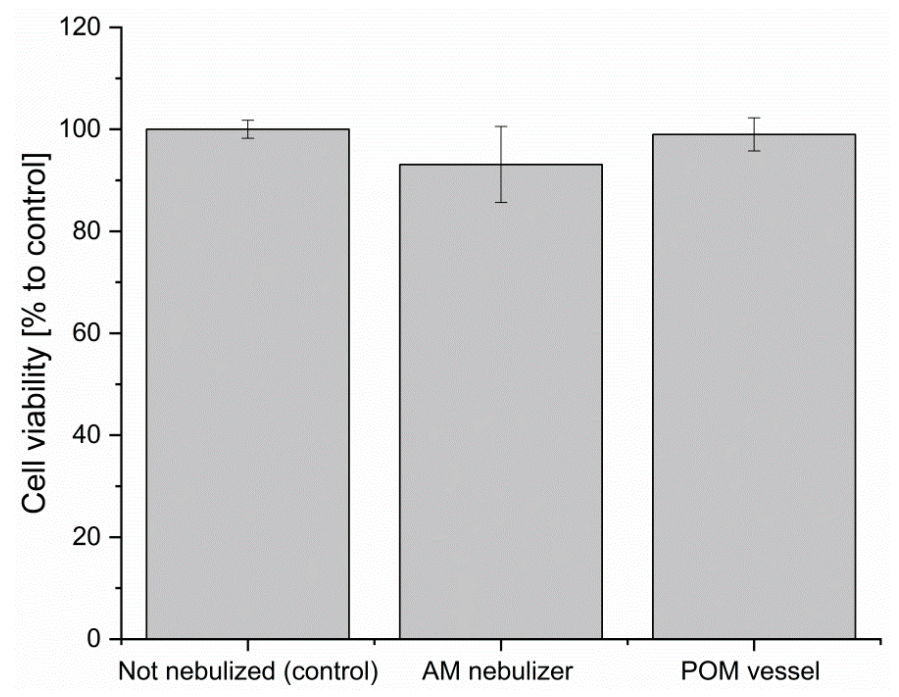

Figure 2. Evaluation of additive manufactured (AM) material used for the manufacture of the LR06. Cell viability of hAD-MSCs in cell culture media that was previously nebulized using: the LR06 (middle column), a vessel made of inert material (POM) (right column). As the control untreated media was used (left column). Cell viability is high (above 90\%) in both experimental set-ups indicating, that no negative effect was generated during the nebulization process. Data points are means and SD for $n=3$. Statistical analysis of the data found no significant difference of shown means (ANOVA One Way, $p=0.24)$.

\subsection{Hydrogel Characterization}

In order to be suitable as biomaterials for 3D bioprinting, hydrogels must have certain properties, as described above. The viscosity of alginate solutions $(\mathrm{Na} / \mathrm{Alg})$ and the storage modulus of crosslinked alginate $(\mathrm{Ca} / \mathrm{Alg})$ were determined for various relevant concentrations.

\subsubsection{Viscosity}

Viscosity of five $\mathrm{Na} / \mathrm{Alg}$ concentrations $(0.5 \%, 1 \%, 2 \%, 3 \%$, and $4 \%(w / v))$ was determined with the help of rheometry. Figure 3 demonstrates the viscosity behavior over a temporal increase of the shear rate. Results show that an increase in alginate concentration leads to a higher material viscosity, going along with the current literature. The lowest concentrations of $0.5-1 \%(w / v)$ alginate show the lowest viscosity of $13.5 \mathrm{mPa}$ (at $1001 / \mathrm{s}$ ) and $23.1 \mathrm{mPa}$ (at $1001 / \mathrm{s})$. The solution with $2 \%(w / v)$ shows a nine-fold higher viscosity of $218.6 \mathrm{mPa}$ (at $1001 / \mathrm{s}$ ) and a further concentration increase results in even higher viscosities: $3 \%(w / v): 1007.7 \mathrm{mPa}$ s (at $1001 / \mathrm{s})$ and $4 \%(w / v): 2156.6 \mathrm{mPa} \mathrm{s}(w / v)$.

Furthermore, the solutions with $2-4 \%$ alginate show a reduction of viscosity at increasing shear rate. This material behavior is typical for some polymers and it is called the shear-thinning effect [41]. Especially for extrusion 3D printing applications, a shear-thinning behavior of the bioink is desired. It leads to a better fluid flow when the material undergoes a certain force, as it is predominant in the extrusion nozzle during 3D bioprinting. Because of the pressure-induced shear stress, the viscosity decreases, which results in easier hydrogel deposition. Once the stress is removed, the viscosity increases again, leading to improved shape stability. In this case, a shear-thinning effect can be observed at alginate concentrations of $2 \%$ and higher. 


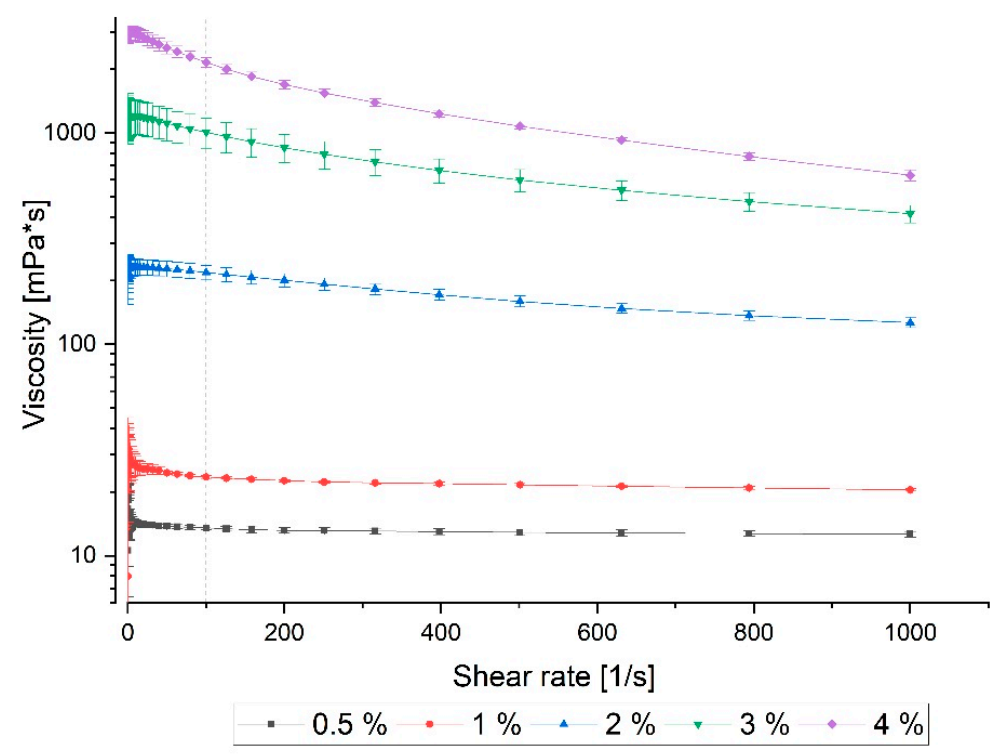

Figure 3. Rheological determination of the viscosity of different sodium alginate concentrations $(\%(w / v))$. Data was obtained by rotational viscometry at $37^{\circ} \mathrm{C}$ and logarithmic increase of the shear rate. $2-4 \%$ samples show a shear-thinning behavior making them suitable for $3 \mathrm{D}$ bioprinting. Data points are means and SD for $n=3$.

\subsubsection{Storage Modulus}

The storage modulus $\left(G^{\prime}\right)$ was determined together with $G^{\prime \prime}$ in oscillation rheology. The $G^{\prime}$ was always higher than the $G^{\prime \prime}$, indicating the all tested constructs behaved like solids. Furthermore, $\mathrm{G}^{\prime}$ stayed at the same level throughout the measurement, indicating that polymerization was complete [41].

Figure 4 shows the storage moduli of different alginate concentrations. As expected and according to the literature, the storage moduli increase with increasing concentration. Whereas, the storage modulus of the lowest alginate concentration $(0.5 \%)$ is about $834 \pm 143 \mathrm{kPa}$, the highest alginate concentration of $4 \%=23.767 \pm 5.614 \mathrm{kPa}$. The other three samples $\mathrm{G}^{\prime}$ values in between this range and vary from $5.738 \pm 2.729$ to $9.144 \pm 3.078 \mathrm{kPa}$.

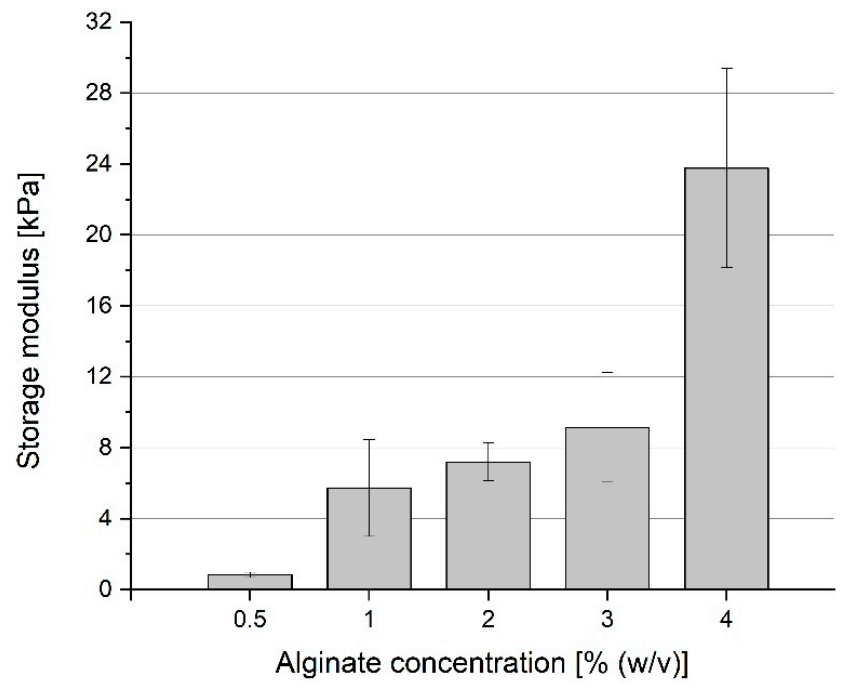

Figure 4. Effect of Cross-linking at five different alginate concentrations on the Storage modulus ( $\left.\mathrm{G}^{\prime}\right)$. $\mathrm{G}^{\prime}$ was determined using model constructs $(2 \mathrm{~cm}$ diameter, $1 \mathrm{~mm}$ height) by performing a time sweep in an oscillation experiment. Data points are means and SD for $n=3$. 


\subsection{Bioprinting}

Before actual bioprinting with stem cells (hAD-MSCs) and murine fibroblasts (NIH-3T3-GFP) was conducted, the bioprinting parameters of used hydrogels were determined (Table 1).

\subsubsection{Bioprinting Parameters and Shape Evaluation}

Table 1 shows the used bioprinting parameters for all experiments. The alginate hydrogels with the lowest concentrations $(0.5 \%$ and $1 \%(w / v))$ did not show shear-thinning properties in rheological experiments. These findings go together with poor resolution and shape fidelity in the printing results in this set-up. These concentrations were not used for further bioprinting with cells (Table 1).

In Figures 5 and 6 printing results with different alginate concentrations containing cells are shown. The printing line diameter averages at $500 \mu \mathrm{m}$. The adherence on the surface was strong enough to let the constructs stick to the petri dish surface during cultivation. The constructs' theoretical side length (please see supplementary material, Figure S1) was supposed to be $9 \mathrm{~mm}$ with the inner lines being $5 \mathrm{~mm}$ long. Actual results show $11 \mathrm{~mm}$ sides and a $6.5 \mathrm{~mm}$ inner line length. Bioprinting of non-linear geometries can be critical and it is often avoided. Figure 6 (bottom right) also shows that narrow curves were manufactured without loss in shape quality or material diffluence.

Table 1. Bioprinting parameters for different $\mathrm{Na} / \mathrm{Alg}$ hydrogel concentrations.

\begin{tabular}{|c|c|c|c|c|c|}
\hline \multirow{2}{*}{$\mathrm{Na} / \mathrm{Alg}[\%,(w / v)]$} & \multirow{2}{*}{$\begin{array}{c}\text { Printing } \\
\text { Temperature }\end{array}$} & \multirow{2}{*}{$\begin{array}{l}\text { Printing Platform } \\
\text { Temperature }\end{array}$} & \multicolumn{2}{|c|}{ Air Pressure for Extrusion } & \multirow{2}{*}{$\mathrm{CaCl}_{2}[\mathrm{c}]$} \\
\hline & & & [PSI] & {$[\mathrm{mPa}]$} & \\
\hline 0.5 & \multirow{5}{*}{$37^{\circ} \mathrm{C}$} & \multirow{5}{*}{$\begin{array}{l}\mathrm{RT} \text { (up to } 40^{\circ} \mathrm{C} \\
\text { possible) }\end{array}$} & $\mathrm{N} / \mathrm{A}$ & $\mathrm{N} / \mathrm{A}$ & \multirow{5}{*}{$500 \mathrm{mM}$} \\
\hline 1 & & & $<1.5$ & $<10.3$ & \\
\hline 2 & & & $1.8-2.4$ & $12.4-16.5$ & \\
\hline 3 & & & $2.1-2.8$ & $14.5-19.3$ & \\
\hline 4 & & & $9.9-13.1$ & $68.2-90.3$ & \\
\hline
\end{tabular}

\begin{tabular}{l|l} 
Live/Dead staining $\quad$ Transmitted-light microscopy
\end{tabular}

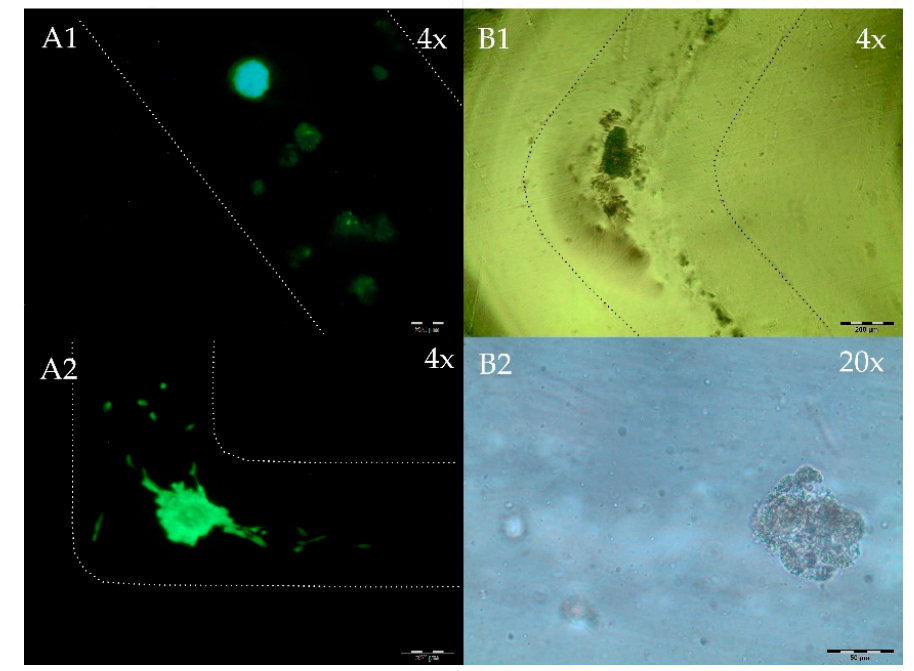

Figure 5. Microscopic images of human adipose tissue-derived mesenchymal stem cells (hAD-MSC) spheroids in $\mathrm{Ca} / \mathrm{Alg}$ hydrogel. Images were taken right after three-dimensional (3D)-bioprint using the LR06. All images show different sections. The borders of the lanes were marked for better visibility. (A) Live/dead (Calcein AM/Propidium Red) images in $4 \times$ magnification (scale bar $=200 \mu \mathrm{m}$ ). In subfigures (A1) and (A2) the majority of the cells show green fluorescence and is viable. (B) Transmitted-light microscopy showing one corner of the $3 \mathrm{D}$ printed construct indicating the precision of the method $((\mathbf{B} 1), 4 \times)$ and a zoom on one spheroid $((\mathbf{B} 2), 20 \times$, scale bar $=50 \mu \mathrm{m})$. Spheroid diameter is $50-200 \mu \mathrm{m}$. 


\subsubsection{HAD-MSC Bioprinting}

Bioprinting with stem cells obtained the best results using $3 \% \mathrm{Ca} / \mathrm{Alg}$ concentration-shown in Figure 5. Average printing line diameter is $400 \mu \mathrm{m}$. A critical area in terms of product quality are corners in the printed object. Figure 5 (top right, bottom left) shows that this area could be manufactured without diffluence of the bioink. The images were taken after $24 \mathrm{~h}$ cultivation time and show both cell types after live/dead staining. The majority of the cells are still viable and survived both the bioprinting process intself and $\mathrm{CaCl}_{2}$ mist-induced bioink polymerization. The majority the of cells in the live/dead image show green fluorescence and are viable.

\subsubsection{NIH-3T3 3D Bioprinting}

In addition to the printed stem cells that are presented above, murine fibrobasts were 3D bioprinted while using the LR06. The used cell line was transduced with a green fluorencence protein. This allows for observing the cells under fluorescent light and track cell viability via GFP protein biosynthesis. The printing results for three different $\mathrm{Ca} / \mathrm{Alg}$ concentrations are presented in Figure 6. The fibroblasts' green appearance is originated in GFP expression in the hydrogel in all 3D bioprinted alginate concentrations, indicating viable cells.

Fluorescence images Transmitted-light microscopy

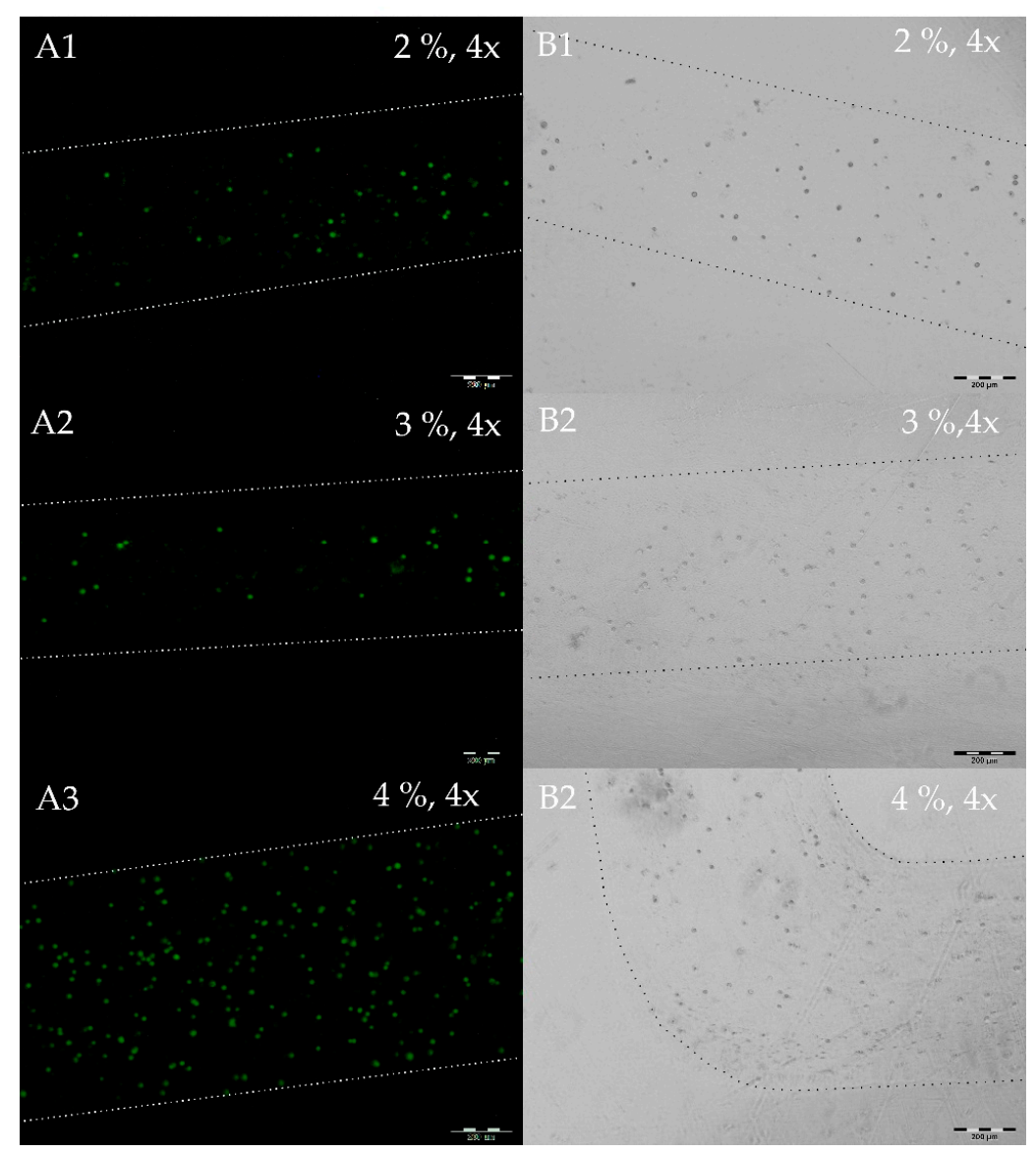

Figure 6. Microscopic images of NIH-3T3-GFP fibroblasts of different alginate-concentrations (2\%, $3 \%$ and $4 \%(v / w)$ ) in $4 \times$ magnification (scale bar $=200 \mu \mathrm{m}$ ). The images were taken after 3D-bioprint using the LR06 and $24 \mathrm{~h}$ cultivation. All images show different sections. The borders of the lanes were marked for better visibility. (A) Fluorescence microscopy images. (A1-A3) show green fibroblasts due to GFP expression. (B) Transmitted-light microscopy images. (B1-B3) show the transmitted-light microscopy images. (B3) shows a $90^{\circ}$ angle of the 3D-bioprinted structure. 


\section{Discussion and Conclusions}

Recent research demonstrates the importance of $\mathrm{Na} / \mathrm{Alg} 3 \mathrm{D}$ bioprinting and its many applications [19,23-29]. The here introduced 3D printed nebulizer (LR06) is an innovative approach of $\mathrm{Na} / \mathrm{Alg}$ 3D bioprinting and it is realized by combining two additive manufacturing methods: (1) The presented nebulizer LR06 was 3D printed using MJP technology and designed for (2) extrusion based 3D bioprinting applications. We demonstrated that this set-up is suitable for manufacturing alginate constructs. This allows the assumption that its application in regulated fields with high standards, such as the biotechnological sector and tissue engineering, is possible. No negative effects on investigated cell lines that came in contact with the generated mist could be measured. The used cells showed no reduction in cell viability compared to the control. In actual application of the LR06, however, no media but $\mathrm{CaCl}_{2}$ gets nebulized and is washed off immediately after crosslinking, minimizing the risk of toxic effects.

Besides the development of LR06, the characterization of suitable alginate concentration for bioprinting was carried out. Viscosity and storage modulus $\left(\mathrm{G}^{\prime}\right.$, describing the behavior of a visco-elastic body under oscillatory shear stress) of $\mathrm{Na} / \mathrm{Alg}$, and $\mathrm{Ca} / \mathrm{Alg}$ in concentrations of $0.5-4 \%$ $(w / v)$ were determined. Corresponding to existing literature, we showed that concentrations of $2 \%$ and higher have shear-thinning material properties, a relevant characteristic of a biomaterial for extrusion bioprinting [42]: The pneumatically applied pressure on the bioink improves its fluid flow behavior and it enhances the construct to hold its shape after extrusion process and before completion of the polymerization. Storage modulus $\left(G^{\prime}\right)$ of the alginate was determined for the same alginate concentrations and an increase with higher $\mathrm{Ca} / \mathrm{Alg}$ concentration could be evaluated. This leads to two conclusions: (1) Lower concentrated $\mathrm{Ca} / \mathrm{Alg}$ results in less stable hydrogels using the LR06 and (2) with each concentration another potential application comes along, some tissues (e.g., vascular tissues) demand stiffer environments than others (e.g., muscle cells). We obtained the best printing results using 3\% and $4 \% \mathrm{Na} / \mathrm{Alg}$. These concentrations are commonly used and described in the literature $[11,23,25,43-46]$. Other groups use composites in order to yield stable products $[10,23]$ or complex protocols (e.g., cryogenic 3D printing [17] or FRESH [32]). With our approach, we were able to produce stable $\mathrm{Na} / \mathrm{Alg}$ structures presenting a feasible method. In addition to that, we could use medium to low concentrated $\mathrm{CaCl}_{2}$ solution, as compared to other studies $[19,27,33]$.

The objective of this work was to demonstrate the application of this innovative printing set-up to the materials of different viscosities. In further studies, direct comparison of the printed structures of the same architecture and volume but different viscosities must be performed. Furthermore, bioprinting with two different cell types (hAD-MSCs spheroids and NIH-3T3-GFP) could be accomplished using the LR06. Cells stayed viable for the cultivation period of at least $24 \mathrm{~h}$ and the printed constructs neither lost their shape nor dissolved in the media. This proof of principle shows that the LR06 works. In order to characterize the device and the 3D bioprinted products thoroughly, further studies need to be performed. The direct comparison of the printed structures of the same architecture and volume but different viscosities, the shape fidelity (mechanical characterization) over time, as well as the warping and adhesion behavior, need to be evaluated. In addition to that, more sophisticated alginate based hydrogel compositions could be tested with the LR06. For example, the use of cell culture medium instead of deionized water for $\mathrm{Na}$ / Alg preparation, which could enhance cell growth and viability [25].

Altogether, the LR06 has proven to be a functional device for sodium alginate bioprinting and it could be used by other researchers working with alginate-based bioinks. In addition, $\mathrm{CaCl}_{2}$ mist is suitable to induce polymerization without a negative impact on the cell containing bioink.

\section{Materials and Methods}

\subsection{Cell Culture}

Human adipose tissue-derived mesenchymal stem cells (hAD-MSCs) were isolated from the adipose tissues of patients after abdominoplastic surgery. Informed consent was obtained from all 
donors and the process was approved by the ethics committee of Hannover Medical School. hAD-MSCs were maintained in alpha-MEM (ThermoFisher Corporation, Waltham, MA, USA) supplemented with 10\% human serum (HS) (CC Pro GmbH, Oberdorla, Germany) and 0.5\% Gentamycin (Biochrom $\mathrm{GmbH}$, Berlin, Germany). All the cells were incubated at $37 \pm 1{ }^{\circ} \mathrm{C}$ in a humidified atmosphere of $5 \% \pm 1 \%(v / v) \mathrm{CO}_{2}$ in air. The cell number in the trypsinized cell suspension was determined using a haemocytometer.

NIH-3T3 mouse fibroblast cells transduced with a GFP were kindly provided by Prof. Andrea Hoffmann (Hannover Medical School). NIH-3T3 were cultivated in Dulbecco's Modified Eagle's Medium (DMEM) (D7777 Sigma-Aldrich, Steinheim, Germany) supplemented with $10 \%$ fetal calf serum (FCS) and $100 \mu \mathrm{g} / \mathrm{mL}$ antibiotics (penicillin-streptomycin) in a humidified environment at $37^{\circ} \mathrm{C}$ and $5 \% \mathrm{CO}_{2}$. Only cells of up to ten passages were used in all experiments.

hAD-MSCs Spheroids

hAD-MSC-spheroids were created using CELLSTAR ${ }^{\circledR}$ Cell-Repellent Surface cultivation flasks (Greiner Bio-One, Frickenhausen, Deutschland). Cell suspension, eight million cells in $10 \mathrm{~mL}$ alpha-MEM (ThermoFisher Corporation, Waltham, MA, USA) supplemented with 10\% human serum (HS) (CC Pro GmbH, Oberdorla, Germany) and 0.5\% Gentamycin (Biochrom GmbH, Berlin, Germany) was placed into T75 flask for $24 \mathrm{~h}$ and the resulting spheroids were centrifuged at $300 \mathrm{~g}$ and resuspended in alginates.

\subsection{Cell Staining (Live/Dead Assay)}

For live/dead staining, bioprinted constructs were incubated for $15 \mathrm{~min}$ at $37^{\circ} \mathrm{C}$ in cell culture medium containing $15 \mu \mathrm{L}$ Calcein-AM (SigmaAldrich Chemie GmbH, München, Deutschland) and $3 \mu \mathrm{L}$ propidium iodide (SigmaAldrick Corporation, St. Louis, MO, USA) per $1.482 \mathrm{~mL}$ alpha-MEM medium. Micrsocopic images were taken with fluorenscence microscope Olympus IX 50 (Olympus Corporation, Tokyo, Japan) with the camera (Olympus SC30, IX-TVAD, Tokyo, Japan) and CellSens Software (CellSens Standard 1.7.1, Olympus).

\subsection{Cell Toxicity Evaluation of Nebulized Media and CellTiterBlue ${ }^{\circledR}$ Assay}

Cell toxicity experiment evaluation is based on the ISO 10993-12:2012 (Biological evaluation of medical devices-Part 12: Sample preparation and reference materials) protocoll. Here, a reduction of cell viability after treatment with extraction media of $>20 \%$ indicates a toxic effect on the cells. The operation procedure for the ISO protocol-derived assay is as follows: $20 \mu \mathrm{L}$ media were nebulized in the respective vessel for $1 \mathrm{~min}$ and was used for the cultivation of AD-MSCs (100 $\mu \mathrm{L}$ and 8000 cells per well) in 96 well plates for $24 \mathrm{~h}$ at $37 \pm 1{ }^{\circ} \mathrm{C}$ in a humidified atmosphere of $5 \% \pm 1 \%(v / v) \mathrm{CO}_{2}$ in air. After medium replacement with $\mathrm{CTB}^{\circledR}$ solution cell culture medium (without nebulization) was incubated for 72 under the same conditions served as control.

CellTiter-Blue ${ }^{\circledR}$ Cell Viability Assay (Promega Corporation, Fitchberg, WI, USA) was conducted according to manufacturer instructions: Briefly, working solution of CellTiter-Blue ${ }^{\circledR}$-reagent in DMEM/alpha-MEM without antibiotics and serum was prepared: $10 \%$ CellTiterBlue ${ }^{\circledR}$-reagent in DMEM or alpha-MEM. $100 \mu \mathrm{L}$ of $\mathrm{CTB}^{\circledR}$-working solution was added into each well. Additionally, two wells without cells were filled with CellTiter-Blue ${ }^{\circledR}$ working solution for the blank measurement. Plates were incubated at $37 \pm 1{ }^{\circ} \mathrm{C}$ in a humidified atmosphere of $5 \% \pm 1 \%(v / v) \mathrm{CO}_{2}$ in air for $105 \mathrm{~min}$. Fluorescence was measured with a microplate fluorometer (Ex579 nm/Em584 nm) (Fluoroskan Ascent, Thermo Electron Corporation, Waltham, MA, USA). 


\subsection{CAD Modeling and 3D Printing Techniques}

\subsubsection{Nebulizer}

The nebulizer used in this study is a custom design. It is assembled of two parts: (1) 3D printed bioprinter-mount with mist chamber and (2) the nebulizer. The latter is a waterproof ultrasonic mist generator (Sens humidity $12 \mathrm{~V}$ nebulizer mod, NB80E-01-H, TDK Corporation, Tokyo, Japan). The settings of the piezo element were set once and used for all presented results: $12 \mathrm{~V}$ operating voltage, $40 \mathrm{~mm} \mathrm{CaCl}$ solution level, $1600 \mathrm{~Hz}$. Please see Figure 1 for further details.

For design, all 3D printed parts were constructed with computer-aided-design (CAD) software Autodesk Inventor Professional 2015 (Autodesk Inc., San Rafael, CA, USA). The nebulizer is designed to be mounted in an Allevi Biobot 1 bioprinter. As rapid prototyping for preliminary experiments (fitting to Biobot 1 bioprinter, $\mathrm{CaCl}_{2}$ mist tests), the fused deposition modeling (FDM) 3D printing technique was used. Therefore, the nebulizer was manufactured with a Makerbot Z18 (Stratasys, Eden Prairie, MN, USA), as printing material poly lactic acid (PLA) was deployed (printing time $14 \mathrm{~h}$ at $0.2 \mathrm{~mm}$ layer height). The final product, which was used for bioprinting experiments, was 3D printed using a high definition multi jet 3D printer (MJP) ProJet 2500 (3D Systems, Rock Hill, SC, USA) and sliced with 3D Sprint software (3D Systems; Rock Hill, SC, USA). Printing material was VisiJet M2-RCL [4] (printing time $21 \mathrm{~h}$ at $30 \mu \mathrm{m}$ layer height). The used 3D printing material was tested both on its chemical stability and biocompatibility in previous studies and published by Raddatz et al. in 2017 [4].

\subsubsection{Bioink Preperation}

As bioink $0.5 \%, 1 \%, 2 \%, 3 \%$ and $4 \%(w / v)$ sodium alginate solution $\left(0.5 ; 1 ; 2,3\right.$, and $4 \mathrm{~g} \mathrm{CaCl}_{2}$ in $100 \mathrm{~mL}$ deionized water) was used and heat steam sterilised before further application. After cultivation as described above, the cells (hAD-MSCs, NIH-3T3-GFP) were centrifuged ( $5 \mathrm{~min}, 300 \times \mathrm{g}$ ) and resuspended in the respective hydrogel concentration $\left(5 \times 10^{6}\right.$ cells $\left./ \mathrm{mL}\right)$. Before centrifugation, NIH-3T3-GFP were detached from the cell culture surface using $4 \mathrm{~mL}$ Trypsin solution. Finally, the bioink was transferred to $10 \mathrm{~mL}$ syringes and instantly mounted in the bioprinter and used for bioprinting.

\subsubsection{D Bioprinting}

3D bioprinted constructs were designed using CAD software mentioned in Section 4.4 and sliced with Repetier Host. For 3D bioprinting, the robotic dispensing bioprinter Biobot 1 (Allevi Inc., Philadelphia, PA, USA) was used.

Bioprinting took plase in petri dishes (Greiner Bio-One International $\mathrm{GmbH}$, Kremsmünster, Österreich). Therefore, the nebulizer was mounted on the bioprinter's building platform, as seen in Figure 1B. In order to improve surface hydrophilicity and gain better printing results, the surface was roughened using abrasive paper (3M Lapping Film Bogen x261, $3 \mu \mathrm{m}$ grain size, 3M Deutschland $\mathrm{GmbH}$, Neuss, Germany). Different printing nozzles were tested and $0.256 \mathrm{~mm}$ diameter nozzles (Nordson Corporation, Westlake, OH, USA) turned out to yield the best results (not shown). Gelation was induced by nebulized $500 \mathrm{mM} \mathrm{CaCl}_{2}$. Further printing parameters are listed in Table 1 .

Before bioprinting all devices and material were sterilized. The bioprinter, petri dishes, and the $3 \mathrm{D}$ printed nebulizer were sterilized prior to the experiment (rinsed with $70 \%(v / v)$ isopropanol and 30 min under UV radiation). $\mathrm{CaCl}_{2}$ solution was sterilized using sterile filtration. Assembly of the nebulizer and actual bioprinting were conducted in sterile environments in a laminar flow cabinet.

Bioprinting work flow: After assembly of the nebulizer and its mounting in the bioprinter, the syringe is filled with hydrogel (containing the hAD-MSC spheroids and NIH-3T3-GFP, respectively). After the 3D print has ended, the construct is exposed to the $\mathrm{CaCl}_{2}$ mist for $30 \mathrm{~s}$. Afterwards, the petri dish with the bioprinted construct is washed with PBS twice and $250 \mu \mathrm{L}$ cell culture media (alpha-MEM) and then incubated at $37 \pm 1{ }^{\circ} \mathrm{C}$ in a humidified atmosphere of $5 \% \pm 1 \%(v / v) \mathrm{CO}_{2}$ in air. 


\subsection{Rheology}

Alginate solutions for rheology experiments were prepared as described in Section 4.4.2. Measurements were performed with a MCR302 (Anton Paar GmbH, Graz, Austria) rheometer equipped with plate-plate geometry and a temperature-controlled peltier element. Each concentration was measured in three replicates.

\subsubsection{Viscosity}

Viscosity measurements were performed with rotational viscometry with a measuring plate of $40 \mathrm{~mm}$ diameter and a gap size of $1 \mathrm{~mm}$. The sample $(1.4 \mathrm{~mL})$ was pipetted onto the measuring plate and was allowed to equilibrate for $2 \mathrm{~min}$ at $37^{\circ} \mathrm{C}$. Measurements were performed at $37^{\circ} \mathrm{C}$ with a logarithmic increase of the shear rate $[1 / \mathrm{s}]$ at 41 time points $0-10001 / \mathrm{s}$.

\subsubsection{Storage Modulus}

The storage modulus was determined for model constructs of $\mathrm{Ca} / \mathrm{Alg}$ discs that were fabricated in the dimensions matching the plate geometry of the rheological instrument. For this purpose, $\mathrm{Na} / \mathrm{Alg}$ solution of appropriate concentration was poured in silicone casting molds $(2 \mathrm{~cm}$ diameter, $1 \mathrm{~mm}$ height). The constructs could not be crosslinked by the nebulizer as the height of $1 \mathrm{~mm}$ provided too high of a diffusion barrier and the solution remained liquid. Therefore, direct addition of $\mathrm{CaCl}_{2}$ solution had to be performed.

Storage modulus measurements were performed wih a time sweep in an oscillation experiment with constant $0.3 \%$ (amplitude gamma) and a frequency of $1 \mathrm{~Hz}$ in the linear viscoelastic range of the alginate hydrogel. Measurements were performed at $37^{\circ} \mathrm{C}$ with a measuring plate of $20 \mathrm{~mm}$, a gap size of $1 \mathrm{~mm}$, and a constant load of $1 \mathrm{~N}$. Values shown are averages of six individual measurements.

\subsection{Chemicals}

Used bulk chemicals were purchased from Sigma-Aldrich Corp. (Taufkirchen, Germany). Deionized water was prepared with Arium ${ }^{\circledR}$ (Sartorius Stedim Biotech GmbH, Göttingen, Germany). Sodium alginate (A2033, 100 kDa, M/G ratio: 1.56) was purchased at Merck KGaA (Darmstadt, Germany).

\subsection{Statistical Analysis}

For the statistical analysis, ANOVA (One Way, Bonferroni) was used. The confidence interval is $99 \%$. As operating software Origin 2018 Version 95G (Origin LabCorporation, Northampton, MA, USA) was used. All data represented as mean \pm SD for measurements for each sample.

Supplementary Materials: The following are available online at http:/ / www.mdpi.com/2079-4983/9/4/63/s1, File S1: LR06-Chamber, File S2: LR06-Lid, Video S1: Demonstration-Video, Figure S1: Object-processing.

Author Contributions: Conceptualization, L.R. and A.L.; Formal analysis, L.R.; Funding acquisition, J.B., D.G., T.B., T.S. and S.B.; Investigation, L.R., A.L. and I.P.; Methodology, L.R.; Project administration, T.B., T.S. and S.B.; Software, L.R.; Supervision, D.G., T.B., T.S. and S.B.; Visualization, L.R.; Writing-original draft, L.R.; Writing-review \& editing, L.R., A.L. and T.S.

Funding: The publication of this article was funded by the Open Access fund of Leibniz Universität Hannover.

Acknowledgments: The authors acknowledge Ethan Overfelt for proofreading the manuscript and all associates for helpful discussions and technical support.

Conflicts of Interest: The authors declare no conflict of interest.

\section{References}

1. Lehmann-Haupt, H.E. Johannes Gutenberg. German Printer. Britannica.com. Available online: https: / / www.britannica.com/biography/Johannes-Gutenberg (accessed on 3 May 2018).

2. Johnston, G. Preaching to a Postmodern World: A Guide to Reaching Twenty-First Century Listeners; Baker Books: Ada, MI, USA, 2001; ISBN 0801063671. 
3. Raddatz, L.; Austerjost, J.; Beutel, S. 3D-Druck: Chancen, Möglichkeiten, Risiken. Chem. Unserer Zeit 2017. [CrossRef]

4. Raddatz, L.; Vries, I.; Austerjost, J.; Lavrentieva, A.; Geier, D.; Becker, T.; Beutel, S.; Scheper, T. Additive manufactured customizable labware for biotechnological purposes. Eng. Life Sci. 2017, 17, 931-939. [CrossRef]

5. Lücking, T.H.; Sambale, F.; Beutel, S.; Scheper, T. 3D-printed individual labware in biosciences by rapid prototyping: A proof of principle. Eng. Life Sci. 2015, 15, 51-56. [CrossRef]

6. Lücking, T.H.; Sambale, F.; Schnaars, B.; Bulnes-Abundis, D.; Beutel, S.; Scheper, T. 3D-printed individual labware in biosciences by rapid prototyping: In vitro biocompatibility and applications for eukaryotic cell cultures. Eng. Life Sci. 2015, 15, 57-64. [CrossRef]

7. Ude, C.; Hentrop, T.; Lindner, P.; Lücking, T.H.; Scheper, T.; Beutel, S. New perspectives in shake flask pH control using a 3D-printed control unit based on $\mathrm{pH}$ online measurement. Sensors Actuators B Chem. 2015, 221, 1035-1043. [CrossRef]

8. Maltman, D.J.; Przyborski, S.A. Developments in three-dimensional cell culture technology aimed at improving the accuracy of in vitro analyses. Biochem. Soc. Trans. 2010, 38, 1072-1075. [CrossRef] [PubMed]

9. Holmes, A.M.; Charlton, A.; Derby, B.; Ewart, L.; Scott, A.; Shu, W. Rising to the challenge: Applying biofabrication approaches for better drug and chemical product development. Biofabrication 2017, 9. [CrossRef] [PubMed]

10. Hölzl, K.; Lin, S.; Tytgat, L.; Van Vlierberghe, S.; Gu, L.; Ovsianikov, A. Bioink properties before, during and after 3D bioprinting. Biofabrication 2016, 8, 032002. [CrossRef] [PubMed]

11. Malda, J.; Visser, J.; Melchels, F.P.; Jüngst, T.; Hennink, W.E.; Dhert, W.J.A.; Groll, J.; Hutmacher, D.W. 25th anniversary article: Engineering hydrogels for biofabrication. Adv. Mater. 2013, 25, 5011-5028. [CrossRef] [PubMed]

12. Koch, L.; Gruene, M.; Unger, C.; Chichkov, B. Laser Assisted Cell Printing. Curr. Pharm. Biotechnol. 2013, 14, 91-97. [CrossRef] [PubMed]

13. Gungor-Ozkerim, P.S.; Inci, I.; Zhang, Y.S.; Khademhosseini, A.; Dokmeci, M.R. Bioinks for 3D bioprinting: an overview. Biomater. Sci. 2018, 6, 915-946. [CrossRef] [PubMed]

14. Derby, B. Printing and prototyping of tissues and scaffolds. Science 2012, 338, 921-926. [CrossRef] [PubMed]

15. Ferris, C.J.; Gilmore, K.G.; Wallace, G.G.; in het Panhuis, M. Biofabrication: An overview of the approaches used for printing of living cells. Appl. Microbiol. Biotechnol. 2013, 97, 4243-4258. [CrossRef] [PubMed]

16. De Mori, A.; Peña Fernández, M.; Blunn, G.; Tozzi, G.; Roldo, M.; De Mori, A.; Peña Fernández, M.; Blunn, G.; Tozzi, G.; Roldo, M. 3D Printing and Electrospinning of Composite Hydrogels for Cartilage and Bone Tissue Engineering. Polymers 2018, 10, 285. [CrossRef]

17. Tan, Z.; Parisi, C.; Di Silvio, L.; Dini, D.; Forte, A.E. Cryogenic 3D Printing of Super Soft Hydrogels. Sci. Rep. 2017, 7, 16293. [CrossRef] [PubMed]

18. Mohanty, S.; Alm, M.; Hemmingsen, M.; Dolatshahi-Pirouz, A.; Trifol, J.; Thomsen, P.; Dufva, M.; Wolff, A.; Emnéus, J. 3D Printed Silicone-Hydrogel Scaffold with Enhanced Physicochemical Properties. Biomacromolecules 2016, 17, 1321-1329. [CrossRef] [PubMed]

19. Duan, B.; Hockaday, L.A.; Kang, K.H.; Butcher, J.T. 3D Bioprinting of heterogeneous aortic valve conduits with alginate/gelatin hydrogels. J. Biomed. Mater. Res. Part A 2013, 101A, 1255-1264. [CrossRef] [PubMed]

20. Shepherd, B.R.; Presnell, S.C.; Evinger, A.J. Engineered Three-Dimensional Connective Tissue Constructs and Methods of Making the Same. WO2013192290A1, 27 December 2013.

21. Lipton, J.I.; Cutler, M.; Nigl, F.; Cohen, D.; Lipson, H. Additive manufacturing for the food industry. Trends Food Sci. Technol. 2015, 43, 114-123. [CrossRef]

22. Jammalamadaka, U.; Tappa, K. Recent Advances in Biomaterials for 3D Printing and Tissue Engineering. J. Funct. Biomater. 2018, 9, 22. [CrossRef] [PubMed]

23. Axpe, E.; Oyen, M.L. Applications of Alginate-Based Bioinks in 3D Bioprinting. Int. J. Mol. Sci. 2016, 17, 1976. [CrossRef] [PubMed]

24. Kim, Y.; Kang, K.; Jeong, J.; Paik, S.S.; Kim, J.S.; Park, S.A.; Kim, W.D.; Park, J.; Choi, D. Three-dimensional (3D) printing of mouse primary hepatocytes to generate 3D hepatic structure. Ann. Surg. Treat. Res. 2017, 92, 67. [CrossRef] [PubMed]

25. Jia, J.; Richards, D.J.; Pollard, S.; Tan, Y.; Rodriguez, J.; Visconti, R.P.; Trusk, T.C.; Yost, M.J.; Yao, H.; Markwald, R.R.; Mei, Y. Engineering alginate as bioink for bioprinting. Acta Biomater. 2014, 10, 4323-4331. [CrossRef] [PubMed] 
26. Wu, D.Y.; Meure, S.; Solomon, D. Self-healing polymeric materials: A review of recent developments. Prog. Polym. Sci. 2008, 33, 479-522. [CrossRef]

27. Nguyen, D.; Hägg, D.A.; Forsman, A.; Ekholm, J.; Nimkingratana, P.; Brantsing, C.; Kalogeropoulos, T.; Zaunz, S.; Concaro, S.; Brittberg, M.; Lindahl, A.; Gatenholm, P.; Enejder, A.; Simonsson, S. Cartilage Tissue Engineering by the 3D Bioprinting of iPS Cells in a Nanocellulose/Alginate Bioink. Sci. Rep. 2017, 7. [CrossRef] [PubMed]

28. Jia, W.; Gungor-Ozkerim, P.S.; Zhang, Y.S.; Yue, K.; Zhu, K.; Liu, W.; Pi, Q.; Byambaa, B.; Dokmeci, M.R.; Shin, S.R.; et al. Direct 3D bioprinting of perfusable vascular constructs using a blend bioink. Biomaterials 2016, 106, 58-68. [CrossRef] [PubMed]

29. Williams, S.K.; Touroo, J.S.; Church, K.H.; Hoying, J.B. Encapsulation of Adipose Stromal Vascular Fraction Cells in Alginate Hydrogel Spheroids Using a Direct-Write Three-Dimensional Printing System. Biores. Open Access 2013, 2, 448-454. [CrossRef] [PubMed]

30. Kyle, S.; Jessop, Z.M.; Al-Sabah, A.; Whitaker, I.S. 'Printability' of Candidate Biomaterials for Extrusion Based 3D Printing: State-of-the-Art. Adv. Healthc. Mater. 2017, 6, 1700264. [CrossRef] [PubMed]

31. Li, Z.; Ramay, H.R.; Hauch, K.D.; Xiao, D.; Zhang, M. Chitosan-alginate hybrid scaffolds for bone tissue engineering. Biomaterials 2005, 26, 3919-3928. [CrossRef] [PubMed]

32. Hinton, T.J.; Jallerat, Q.; Palchesko, R.N.; Park, J.H.; Grodzicki, M.S.; Shue, H.J.; Ramadan, M.H.; Hudson, A.R.; Feinberg, A.W. Three-dimensional printing of complex biological structures by freeform reversible embedding of suspended hydrogels. Sci. Adv. 2015, 1. [CrossRef] [PubMed]

33. George, A.; Hu, B.; Xue, Z.; Du, Z.; Nageswar, Y.V.D.; Murthy, S.N.; Madhav, B.; Shankar, J.; Lin, T.-H.; Huang, W.-H.; et al. Biomineralization and Biomimetic Synthesis of Biomineral and Nanomaterials. In Advances in Biomimetics; InTech: Rijeka, Croatia, 2011; ISBN 978-953-307-191-6.

34. Dreimann, J. Evaluierung und Optimierung Eines Neuartigen Bioreaktorsystems-Technische Informationsbibliothek (TIB); Leibniz Universität Hannover: Hannover, Germany, 2014.

35. Tscheschke, B. Nebelkammerreaktoren zur Kultivierung Biotechnologisch Relevanter-Technische Informationsbibliothek (TIB); Leibniz Universität Hannover: Hannover, Germany, 2015.

36. Barreras, F.; Amaveda, H.; Lozano, A. Transient high-frequency ultrasonic water atomization. Exp. Fluids 2002, 33, 405-413. [CrossRef]

37. Avvaru, B.; Patil, M.N.; Gogate, P.R.; Pandit, A.B. Ultrasonic atomization: Effect of liquid phase properties. Ultrasonics 2006, 44, 146-158. [CrossRef] [PubMed]

38. Wozniak, G. Zerstäubungstechnik; Springer: Berlin/Heidelberg, Germnay, 2003; ISBN 978-3-642-62509-1.

39. Safety Data Sheet According to VisiJet ${ }^{\circledR}$ M2-RCL 1; 3D Systems, Inc.: Rock Hill, SC, USA, 2015.

40. Biological Evaluation of Medical Devices_Part 5: Tests for In Vitro Cytotoxicity; ISO 10993-5:2009; ISO: Geneva, Switzerland, 2009.

41. Mezger, T.G. Angewandte Rheologie-Mit Joe Flow auf der Rheologie-Straße; Anton Paar GmbH: Graz, Austria, 2014; ISBN 978-3-200-03652-9.

42. Wilson, S.A.; Cross, L.M.; Peak, C.W.; Gaharwar, A.K. Shear-Thinning and Thermo-Reversible Nanoengineered Inks for 3D Bioprinting. ACS Appl. Mater. Interfaces 2017, 9, 43449-43458. [CrossRef] [PubMed]

43. Tabriz, A.G.; Hermida, M.A.; Leslie, N.R.; Shu, W. Three-dimensional bioprinting of complex cell laden alginate hydrogel structures. Biofabrication 2015, 9, 45012. [CrossRef] [PubMed]

44. Song, S.-J.; Choi, J.; Park, Y.-D.; Hong, S.; Lee, J.J.; Ahn, C.B.; Choi, H.; Sun, K. Sodium Alginate Hydrogel-Based Bioprinting Using a Novel Multinozzle Bioprinting System. Artif. Organs 2011, 35, 1132-1136. [CrossRef] [PubMed]

45. He, Y.; Yang, F.; Zhao, H.; Gao, Q.; Xia, B.; Fu, J. Research on the printability of hydrogels in 3D bioprinting. Sci. Rep. 2016, 6, 29977. [CrossRef] [PubMed]

46. Zhao, Y.; Li, Y.; Mao, S.; Sun, W.; Yao, R. The influence of printing parameters on cell survival rate and printability in microextrusion-based 3D cell printing technology. Biofabrication 2015, 7, 045002. [CrossRef] [PubMed]

(C) 2018 by the authors. Licensee MDPI, Basel, Switzerland. This article is an open access article distributed under the terms and conditions of the Creative Commons Attribution (CC BY) license (http:/ / creativecommons.org/licenses/by/4.0/). 